Pacific Journal of Mathematics

THE CHARACTERISTIC FUNCTION OF A HARMONIC
FUNCTION IN A LOCALLY EUCLIDEAN SPACE 


\section{THE CHARACTERISTIC FUNCTION OF A HARMONIC FUNCTION IN A LOCALLY EUCLIDEAN SPACE}

\section{WiLLiaM IAEE JOHNSON}

We define the characteristic function for each harmonic function having prescribed singularities in a locally Euclidean space and the class of harmonic functions with bounded characteristic. The main result is that any harmonic function of bounded characteristic can be represented as the difference of two positive harmonic functions with prescribed singularities. Thus the well-known theory of the characteristic functions associated with meromorphic functions has an analogue for harmonic functions in locally Euclidean spaces.

1. Preliminaries.

2. Let $V$ be a locally Euclidean $n$-space $(n>2)$. By definition $V$ is an $n$-manifold for which the defining homeomorphisms $\eta$ of open sets $O$ with $n$-balls of $R^{n}$ are isometries. We shall use the same symbol $z$ for the point of $V$ and for its parametric image. Properties of a function $u(z)$ are always to be understood in terms of the parameter. Expressions such as "an $n$-ball centered at $a$ " and " $|z-a|$, the distance between $z$ and $a$ " refer to the parametric representation.

3. Let $C$ denote the unit ball in $R^{n}$ and $P$ a coordinate hyperplane. A region $G \subset V$ is a bordered region if

(B-1) $B=\partial G$ is compact,

(B-2) for any $z \in B$ there is a neighborhood $N(z)$ and a diffeomorphism $\dot{\phi}$ of $N(z)$ with $C$ such that $\phi(N \cap B)=C \cap P$ and $\phi(N \cap G)$ is one of the two half-balls of $C-P$.

A bordered region $G \subset V$ is regular if

(R-1) $\bar{G}$ is compact,

(R-2) $B=\partial(V-\bar{G})$,

(R-3) all components of $V-G$ are noncompact.

The flux of a function $u \in C^{1}$ on the border $B$ of a bordered region $G$ is

$$
\int_{B}(\partial u / \partial n) d S
$$

where $d S$ is an area element on $B$ and $\partial / \partial n$ is the exterior normal 
derivative.

4. The standard properties of harmonic functions which are true for locally Euclidean spaces are used without qualification. In particular we have Green's formulae, the mean value properties, the Poisson formula, and Harnack's inequality.

The characteristic singularity ([2], p. 241) for a function $u \in H$ in $G-a$ is

$$
s(z)=|z-a|^{2 \sim n} / \omega_{n}(n-2),
$$

where $\omega_{n}$ is the area of the unit sphere in $R^{n}$. The flux of $s(z)$ across a sphere centered at $a$ is -1 .

The capacity function $p_{\theta}$ of $\bar{G}$ with singularity (1) at $a$ has a constant value on $\partial G$ such that the regular part of $p_{G}$ tends to zero at $a$. For $u \in H$ in $G$, we have

$$
u(\alpha)=\int_{\partial \theta} u(z)(\partial p(z, a) / \hat{\partial} n) d S .
$$

2. Harmonic functions of bounded characteristic.

5. Let $L$ denote the class of harmonic functions regular in $V$ except for singularities of the type

$$
\lambda_{j}\left|z-z_{j}\right|^{2-n} / \omega_{n}(n-2), \quad j=1, \cdots, m,
$$

where the $\lambda_{j}$ are real numbers, and the $z_{j}$ are arbitrary points of $V$. The subclass of positive functions in $L$ is denoted by $L P$.

Given a function $h \in L$ in $V$ which is finite at $a \in U$, choose a regular region $\Omega \subset V$ containing $a$. Let $x_{\Omega}+(z)$ be the solution of the Dirichlet problem in $\Omega$ with boundary values $h^{+}=\max (h, 0)$ on $\partial \Omega$. By (2)

$$
x_{\Omega}^{+}(z)=\int_{\partial \Omega} h^{+}(t)\left(\partial p_{\Omega}(t, z) / \partial n\right) d S,
$$

where the capacity function $p_{\Omega}$ has its singularity at $z$. Similarly $x_{\Omega}^{-}(z) \in H$ in $\Omega$ with boundary values $h^{-}=\max (-h, 0)$ on $\partial \Omega$, and

$$
x_{\Omega}^{-}(z)=\int_{\partial \Omega} h^{-}(t)\left(\partial p_{\Omega}(t, z) / \partial n\right) d S .
$$

Let the positive and negative singularities of $h$ be $a_{i}$ and $b_{j}$ respectively, and define

$$
\begin{aligned}
& y_{\Omega}^{+}(z)=\sum_{a_{i} \in \Omega} \lambda_{i} g_{\Omega}\left(z, a_{i}\right), \\
& \lambda_{i}>0, \\
& y_{\Omega}^{-}(z)=\sum_{b_{j} \in \Omega} \lambda_{j} g_{\Omega}\left(z, b_{j}\right) \text {, } \\
& \lambda_{j}<0 \text {; }
\end{aligned}
$$




$$
\begin{aligned}
& u_{\Omega}^{+}(z)=x_{\Omega}^{+}(z)+y_{\Omega}^{+}(z), \\
& u_{\Omega}^{-}(z)=x_{\Omega}^{-}(z)+y_{\Omega}^{-}(z) .
\end{aligned}
$$

Here $g_{\Omega}(z, t)$ is the Green's function of $\Omega$ with singularity at $t$.

We call $C(\Omega, h)=u_{\Omega}^{+}(a)$ the characteristic function of $h$ with respect to $a$ and $\Omega$. The class $L C$ of functions of bounded characteristic consists of $h \in L$ with

$$
C(\Omega, h) \leqq M
$$

for some $M<\infty$ and all $\Omega \subset V$. Note that $C(\Omega,-h)=u_{\Omega}^{-}(a)$. It is a consequence of Theorem 1 below that the class of $L C$ is independent of the point $a$ chosen.

\section{The decomposition theorem.}

6. THeOREm 1. A necessary and sufficient condition for $h \in L C$ in $V$ is that

$$
h=u-v,
$$

where $u, v \in L P$ in $V$.

We first prove that $C(\Omega, h)$ is an increasing function of $\Omega$.

Lemma 1. Let $h \in L$ in $V$ and $\Omega \subset \Omega^{\prime}$ regular subregions of $V$ with $a \in \Omega$. Then

$$
u_{\Omega}{ }^{+}(z) \leqq u_{\Omega^{\prime}}{ }^{+}(z)
$$

Proof. The function $h-y_{\Omega}^{+}+y_{\Omega}^{-} \in C^{0}$ in $\bar{\Omega}, \in H$ in $\Omega$, and has boundary values $h$ on $\partial \Omega$. From (2) we have

$$
h(z)-y_{\Omega}^{+}(z)+y_{\Omega}^{-}(z)=\int_{\partial \Omega} h(t)\left(\partial p_{\Omega}(t, z) / \partial n\right) d S .
$$

Here we assume that no singularity of $h$ lies on $\partial \Omega$. An appeal to a continuity argument will give the same result if this is not the case.

On separating $h$ into $h^{+}$and $h^{-}$, the right side of (8) is $x_{a}^{+}(z)-$ $x_{\Omega}^{-}(z)$. Hence

$$
h(z)=u_{\Omega}^{+}(z)-u_{\Omega}^{-}(z) .
$$

Since $u_{\Omega}^{-}(z) \geqq 0$, (9) gives for all $\Omega \subset V$

$$
h^{+}(z) \leqq u_{\Omega}^{+}(z) .
$$

By virtue of (4), (10), and (2), we have 


$$
\begin{aligned}
x_{\Omega}^{+}(z) & =\int_{\partial \Omega} h^{+}(t)\left(\partial p_{\Omega}(t, z) / \partial n\right) d S \\
& \leqq \int_{\partial \Omega} u_{\Omega^{\prime}}^{+}(t)\left(\partial p_{\Omega}(t, z) / \partial n\right) d S \\
& =\int_{\partial \Omega}\left[u_{\Omega^{\prime}}^{+}(t)-y_{\Omega}^{+}(t)\right]\left(\partial p_{\Omega}(t, z) / \partial n\right) d S \\
& =u_{\Omega^{\prime}}^{+}(z)-y_{\Omega}^{+}(z) .
\end{aligned}
$$

We conclude that $u_{\Omega}^{+}(z) \leqq u_{\Omega^{\prime}}^{+}(z)$.

\section{Lemma 2. If $h \in L C$ in $V$, then $-h \in L C$.}

Proof. Choose $a \in V$ with $h(a) \neq \infty$. The characteristic function of $-h$ is $u_{\Omega}^{-}(a)$. Using (9) with argument $a$ we see that the boundedness of $u_{\Omega}^{+}(\alpha)$ guarantees the same for $u_{\Omega}^{-}(a)$, and $-h \in L C$.

8. Proof of Theorem 1. Let $h \in L C$ in $V$. The function $u_{\Omega}^{+}(z)$, which increases with $\Omega$ by Lemma 1 , is harmonic on $\Omega-\left\{a_{i}\right\}$. The limit function $u(z)$ is either harmonic or $+\infty$ in $V-\left\{a_{i}\right\}$. The former must hold since $u_{\Omega}^{+}(a)$ is bounded for all $\Omega$ by assumption. Analogously, $u_{\Omega}^{-}(z)$ tends to $v(z) \in H$ in $V-\left\{b_{j}\right\}$. Formula (9) implies in the limit that

$$
h(z)=u(z)-v(z)
$$

in $V$, and $u, v \in L P$.

To establish the converse, suppose that $h(z)=u_{1}(z)-v_{1}(z)$ with $u_{1}, v_{1} \in L P$. Since $u_{1}(z) \geqq 0$, (9) implies that all positive singularities of $u_{\Omega}^{+}$are among those of $u_{1}$. Thus $u_{1}-u_{\Omega}^{+}$is superharmonic in $\Omega$ and takes its minimum on $\partial \Omega$. This minimum is nonnegative, and, consequently, $u_{\Omega}^{+}(z) \leqq u_{1}(z)$ in $\Omega$. If $u_{1}(\alpha)$ is finite, then $h \in L C$.

If $u_{1}(a)=\infty$, let $u_{2}(z)=u_{1}(z)-\lambda g_{2}(z, a)$, where $\lambda$ is the order of singularity of $u_{1}$ at $\alpha$. Similarly let $v_{2}(z)=v_{1}(z)-\mu g_{\Omega}(z, \alpha)$, where $\mu$ is the order of the singularity of $v_{2}(z)$ at $a$. Then

$$
h(z)=u_{2}(z)-v_{2}(z)+(\lambda-\mu) g_{\Omega}(z, a) .
$$

If $\lambda \leqq \mu$, then $u_{\Omega}^{+}(a) \leqq u_{2}(a)<\infty$, and $h \in L C$. If $\lambda>\mu$, then $-h \in$ $L C$, and $h \in L C$ by Lemma 2 .

\section{Extremal decomposition.}

9. THEOREM 2. Let $h \in L C$ in $V$ and let $u, v$ be the functions constructed in the proof of Theorem 1. For any decomposition $h=$ $u_{1}-v_{1}$, with $u_{1}, v_{1} \in L P$, we have $u \leqq u_{1}, v \leqq v_{1}$. 
Proof. For $\Omega \subset V$ we have

$$
h(z)=u_{1}(z)-v_{1}(z)=u_{\Omega}^{+}(z)-u_{\Omega}^{-}(z) .
$$

By the reasoning of 8 ,

$$
\begin{aligned}
& u_{\Omega}^{+}(z) \leqq u_{1}(z), \\
& u_{\Omega}^{-}(z) \leqq v_{1}(z)
\end{aligned}
$$

Since the inequalities hold for all $\Omega \subset V$, the limit functions $u$ and $v$ are dominated by $u_{1}$ and $v_{1}$ respectively.

10. Suppose there is an $h \in L P$ in $V$. For any $a \in \Omega \subset V$ the Green's function with singularity at $a$ exists. Since $g_{\Omega}$ vanishes on $\partial \Omega, h-g_{\Omega}$ is superharmonic. The $g_{\Omega}$ increase with $\Omega$, and we conclude that the Green's function $g_{V}$ of $V$ exists.

11. The extremal functions $u$ and $v$ of Theorem 2 have a further decomposition.

Theorem 3. $h \in L C$ in $V$ if and only if

$$
h=\left(x^{+}+y^{+}\right)-\left(x^{-}+y^{-}\right),
$$

where the $x$-functions are regular harmonic in $V$ and

$$
y^{+}=\sum_{a_{i} \in V} \lambda_{i} g_{V}\left(z, a_{i}\right), y^{-}=\sum_{b_{j} \in V} \lambda_{j} g_{V}\left(z, b_{j}\right) .
$$

REMARK. The Green's function for $V$ exists by 10 and will be constructed in the course of the proof.

Proof. If $h$ has the asserted decomposition, then $h \in L C$ by Theorem 1.

Conversely, let $h \in L C$ and choose regular regions $\Omega_{0} \subset \Omega \subset V$. The function $y_{\Omega}^{+}-y_{\Omega_{0}}^{+}$is harmonic in $\Omega_{0}$ and nonnegative on $\partial \Omega_{0}$. By the minimum principle $y_{\Omega_{0}}^{+}(z) \leqq y_{\Omega}^{+}(z)$ in $\Omega_{0}$. Similarly $y_{\Omega_{0}}^{-}(z) \leqq y_{\Omega}^{-}(z)$ in $\Omega_{0}$. Let $y^{+}$and $y^{-}$be the respective limit functions as $\Omega \rightarrow V$. The singularities of $y_{\Omega}^{+}$are among those of $u$, and $y_{\Omega}^{+}(z) \leqq u(z)$ in $\Omega$. The limit function $y^{+}$is therefore harmonic in $V-\left\{a_{i}\right\}$. Also $y^{-} \in H$ in $V-\left\{b_{j}\right\}$.

We show next that $\lim _{\Omega \rightarrow V} y_{\Omega}^{+}(z)=\sum_{a_{i} \in V} \lambda_{i} g_{V}\left(z, a_{i}\right)$. The proof of the corresponding result for $y_{\Omega}^{-}$is similar and will be omitted. We have

$$
\begin{aligned}
y_{\Omega}^{+}(z) & =\sum_{a_{i} \in \Omega} \lambda_{i} g_{\Omega}\left(z, a_{i}\right) \leqq \sum_{a_{i} \in \Omega} \lambda_{i} g_{V}\left(z, a_{i}\right) \\
& \leqq \sum_{a_{i} \in V} \lambda_{i} g_{V}\left(z, a_{i}\right),
\end{aligned}
$$


and consequently

$$
\lim \sup y_{\Omega}^{+}(z) \leqq \sum_{a_{i} \in V} \lambda_{i} g_{V}\left(z, a_{i}\right) .
$$

On the other hand,

$$
\begin{aligned}
\sum_{a_{i} \in \Omega_{0}} \lambda_{i} g_{V}\left(z, a_{i}\right) & =\lim _{\Omega \rightarrow V} \sum_{a_{i} \in \Omega_{0}} \lambda_{i} g_{\Omega}\left(z, a_{i}\right) \\
& \leqq \liminf \sum_{a_{i} \in \Omega} \lambda_{i} g_{\Omega}\left(z, a_{i}\right)=\liminf y_{\Omega}^{+}(z) .
\end{aligned}
$$

On passing to the limit $\Omega_{0} \rightarrow V$ we obtain

$$
\sum_{a_{i} \in V} \lambda_{i} g_{V}\left(z, a_{i}\right) \leqq \lim \inf y_{\Omega}^{+}(z),
$$

and $y^{+}$has the asserted decomposition.

We recall that $x_{\Omega}^{+}=u_{\Omega}^{+}-y_{\Omega}^{+} \rightarrow u-y^{+}$. Since the $x_{\Omega}^{+}$are regular harmonic, it follows that the same is true of the limit function $x^{+}$in $V$. Similarly $x^{-} \in H$ in $V$.

\section{Classification theory.}

12. Given a locally Euclidean $n$-space $V$ and a class of functions $T$ defined in $V$, we say that $V \in O_{T}$ if the only functions of class $T$ in $V$ are constants. This definition has been used in the classification of Riemann surfaces ([1], [3], [4]) and of locally Euclidean spaces [6]. We know [6] that the $O$-classes for $H B$, bounded harmonic functions, and for $H D$, harmonic functions with finite Dirichlet integral, are related by

$$
O_{H B} \subset O_{H D}
$$

Since any bounded function becomes positive by addition of a suitable constant, we also have

$$
O_{H P} \subset O_{H B}
$$

where $H P$ is the class of positive harmonic functions. By definition $V \in O_{G}$ if $V$ has no Green's function.

By considering the classes $L C$ and $L P$ defined in 5 we can incorporate the corresponding $O$-classes into the inclusion chain. We find beginning in 13 that

$$
O_{G} \subset O_{L G} \subset O_{L P} \subset O_{H P} \subset O_{H B} \subset O_{H D} .
$$

The first three classes are in fact equal, and the inclusions

$$
O_{G} \subset O_{H P} \text { and } O_{H P} \subset O_{H B}
$$

are strict. The strictness of the last inclusion in (13) is an open question. 
13. Let $h \in L C$ in $V$. To show that $O_{G} \subset O_{L \sigma}$ we may assume that $h$ has at least one positive singularity at $a \in V$. For any regular $\Omega \subset V$ with $a \in \Omega$, let $g_{\Omega}(z, a)$ be the Green's function of $\Omega$ with singularity at $a$. There exists a decomposition $h=u-v$, where $u, v \in L P$ in $V$. The function $u-g_{\Omega}$ is superharmonic in $\Omega$ and nonnegative on $\partial \Omega$. Hence $g_{\Omega} \leqq u$ throughout $\Omega$. Since $g_{\Omega}$ increases with $\Omega$, we conclude that $g_{V}$ exists and that $O_{G} \subset O_{L \sigma}$.

Since $L P \subset L C$, we have $O_{L \sigma} \subset O_{L P}$. It is clear that $H P \subset L P$ and hence $O_{L P} \subset O_{H P}$.

14. Since $L$ is a class of functions which have singularities of the type $|z-a|^{2-n}$, a space $V$ has $L$ functions if and only if it has a Green's function. The inclusion $O_{G} \subset O_{L P}$ is thus an equality, and the first three 0 -classes in (13) are equal.

15. The inclusion $O_{G} \subset O_{H P}$ is obviously strict. Also $O_{H P}$ is strictly contained in $O_{H B}$, as is easily seen by considering $V=R^{n}-\{0\}$. A single point is a removable singularity for $H B$ functions, an easy consequence of Harnack's inequality. Hence an $H B$ function in $V$ has a harmonic extension to all of $R^{n}$ and must therefore be constant. On the other hand, the Green's function for $R^{n}$ with singularity at 0 is a nonconstant $H P$ function in $V$. Thus $V \in O_{H B}$ and the inclusion $O_{H P} \subset O_{H B}$ is strict.

\section{REFERENCES}

1. L. Ahlfors and L. Sario, Riemann Surfaces, Princeton University Press, 1960.

2. R. Courant and D. Hilbert, Methods of Mathematical Physics, Vol. II, Interscience, New York, 1962.

3. L. Sario, Über Riemannsche Flächer mit hebbarem Rand, Ann. Acad. Sci. Fenn., Ser. A. I. 50 (1948).

4. - Sur la classificatication des surfaces de Riemann, C. R. Unzideme Congr. Math. Scand., Trondheim (1949), 229-238.

5. - On locally meromorphic functions with singlevalued moduli, Pacific J. Math, 13 (1963), 709-724.

6. Classification of locally Euclidean spaces, Nagoya Math. J. 25 (1965), 87111.

Received December 1, 1965, and in revised Form October, 12, 1966. This material is included in the author's doctoral dissertation written at the University of California, Los Angeles. The author is indebted to the Army Research Office (Durham) for support under Contract DA-ARO(D)-31-124-G40 and to Prof. Leo Sario for his guidance and inspiration.

U. S. Naval Ordnance Test Station

China Lake, California 



\section{PACIFIC JOURNAL OF MATHEMATICS}

\section{EDITORS}

\section{H. ROYDEN \\ Stanford University \\ Stanford, California}

\author{
J. P. JANS \\ University of Washington \\ Seattle, Washington 98105
}

J. DugundJI

Department of Mathematics

Rice University

Houston, Texas 77001

RichaRd ARENS

University of California

Los Angeles, California 90024

\section{ASSOCIATE EDITORS}
E. F. BECKENBACH
B. H. NeumanN
F. WOLF
K. YOSIDA

\section{SUPPORTING INSTITUTIONS}

\author{
UNIVERSITY OF BRITISH COLUMBIA \\ CALIFORNIA INSTITUTE OF TECHNOLOGY \\ UNIVERSITY OF CALIFORNIA \\ MONTANA STATE UNIVERSITY \\ UNIVERSITY OF NEVADA \\ NEW MEXICO STATE UNIVERSITY \\ OREGON STATE UNIVERSITY \\ UNIVERSITY OF OREGON \\ OSAKA UNIVERSITY \\ UNIVERSITY OF SOUTHERN CALIFORNIA
}

\author{
STANFORD UNIVERSITY \\ UNIVERSITY OF TOKYO \\ UNIVERSITY OF UTAH \\ WASHINGTON STATE UNIVERSITY \\ UNIVERSITY OF WASHINGTON

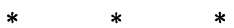 \\ AMERICAN MATHEMATICAL SOCIETY \\ CHEVRON RESEARCH CORPORATION \\ TRW SYSTEMS \\ NAVAL ORDNANCE TEST STATION
}

Mathematical papers intended for publication in the Pacific Journal of Mathematics should be typewritten (double spaced). The first paragraph or two must be capable of being used separately as a synopsis of the entire paper. It should not contain references to the bibliography. Manuscripts may be sent to any one of the four editors. All other communications to the editors should be addressed to the managing editor, Richard Arens at the University of California, Los Angeles, California 90024.

50 reprints per author of each article are furnished free of charge; additional copies may be obtained at cost in multiples of 50 .

The Pacific Journal of Mathematics is published monthly. Effective with Volume 16 the price per volume (3 numbers) is $\$ 8.00$; single issues, $\$ 3.00$. Special price for current issues to individual faculty members of supporting institutions and to individual members of the American Mathematical Society: $\$ 4.00$ per volume; single issues $\$ 1.50$. Back numbers are available.

Subscriptions, orders for back numbers, and changes of address should be sent to Pacific Journal of Mathematics, 103 Highland Boulevard, Berkeley 8, California.

Printed at Kokusai Bunken Insatsusha (International Academic Printing Co., Ltd.), 7-17, Fujimi 2-chome, Chiyoda-ku, Tokyo, Japan.

PUBLISHED BY PACIFIC JOURNAL OF MATHEMATICS, A NON-PROFIT CORPORATION

The Supporting Institutions listed above contribute to the cost of publication of this Journal, but they are not owners or publishers and have no responsibility for its content or policies. 


\section{Pacific Journal of Mathematics}

\section{Vol. 23, No. 2 \\ April, 1967}

Herbert Stanley Bear, Jr. and Bertram John Walsh, Integral kernel for

one-part function spaces .......................... 209

Mario Borelli, Some results on ampleness and divisorial schemes ....... 217

John A. Erdos, Unitary invariants for nests . ................... 229

Nathaniel Grossman, The volume of a totally-geodesic hypersurface in a pinched manifold.................................. 257

D. M. Hyman, A generalization of the Borsuk-Whitehead-Hanner theorem ............................................. 263

I. Martin (Irving) Isaacs, Finite groups with small character degrees and large prime divisors ............................. 273

I. Martin (Irving) Isaacs, Two solvability theorems ................ 281

William Lee Johnson, The characteristic function of a harmonic function in a locally Euclidean space ............................... 291

Ralph David Kopperman, Application of infinitary languages to metric spaces ............................................. 299

John Lauchlin MacDonald, Relative functor representability ............ 311

Mahendra Ganpatrao Nadkarni, A class of measures on the Bohr group.... 321

Keith Lowell Phillips, Hilbert transforms for the p-adic and p-series fields....................................... 329

Norman R. Reilly and Herman Edward Scheiblich, Congruences on regular semigroups ...

Neil William Rickert, Measures whose range is a ball ... . .

Gideon Schwarz, Variations on vector measures

Ronald Cameron Riddell, Spectral concentration for self-adjoint operators. .

Haskell Paul Rosenthal, A characterization of restrictions of

Fourier-Stieltjes transforms ................... 